\title{
PERSISTÊNCIA E MEMÓRIA LONGA SAZONAL NA SÉRIE DE DESEMPREGO DA REGIÃO METROPOLITANA DE SÃO PAULO
}

\author{
Guilherme de O. L. C. Marques \\ Vera Lúcia Fava ${ }^{\dagger}$
}

\begin{abstract}
Resumo
Este artigo aborda o tema da persistência na taxa de desemprego da região metropolitana de São Paulo. Foram utilizados modelos SARFIMA de integração fracionária para avaliar a dinâmica de absorção dos choques econômicos pela série de desemprego. A hipótese de histerese no desemprego foi avaliada usando os modelos de memória longa. Os resultados obtidos pelo arcabouço fracionário foram contrapostos aos do paradigma $I(1)-I(0)$ e revelaram a incapacidade dos modelos tradicionais SARIMA de extrair corretamente o comportamento de baixa frequência da série temporal. Verificou-se que o modelo sazonal tradicional induz à superavaliação da persistência na série de tempo estudada.
\end{abstract}

JEL classification: C22, C32, C15

Palavras-chave: memória longa sazonal, integração fracionária e histerese.

\begin{abstract}
This paper deals with the persistence theme in the unemployment rate of São Paulo metropolitan region. SARFIMA fractional integration models were used to evaluate the dynamics of economic shock absorption by the unemployment series. The hysteresis hypothesis in the unemployment rate was evaluated using long-memory models. The results found using the fractional framework were compared with those of the $I(1)-I(0)$ paradigm, and they showed the inability of the traditional SARIMA models to correctly extract the low-frequency behavior of the time series. It was shown that traditional seasonal model leads to an overvaluation of the persistence in the series.
\end{abstract}

JEL classification: C22, C32, C15

Palavras-chave: seasonal long memory, fractional integration and hysteresis.

\footnotetext{
* Universidade Federal do ABC

${ }^{\dagger}$ Universidade de São Paulo
} 


\section{Introdução}

Existem fatores econômicos cuja avaliação em prol de políticas públicas pressupõe o nível agregado dos dados e em amplos intervalos de tempo. Um desses casos é a noção de rigidez no mercado de trabalho que, entre outros fatores, define as bases técnicas para o seguro desemprego. A identificação e mensuração do grau de rigidez no mercado de trabalho será o tema explorado nesse trabalho por meio da análise estatística de processos estocásticos fracionariamente integrados.

A Teoria Econômica percebe nas instituições uma das principais fontes da elevada rigidez no mercado de trabalho. Zylberstajn \& Balbinoto Neto (1999), por exemplo, comentam que a persistência nas taxas de desemprego está associada a aspectos institucionais que não incentivam a contratação de novos trabalhadores ou de trabalhadores desempregados. Ulissea $(2008,2010)$ o autor estudou a relação entre as instituições brasileiras e a decisão do trabalhador de ingressar no mercado formal ou informal da economia. O autor desenvolveu um modelo de "macthing" com dois setores sobre o qual realizou simulações do efeito de diferentes políticas econômicas visando reduzir a informalidade e aumentar a performance no mercado de trabalho brasileiro. Os estudos mostram uma alta sensibilidade do desemprego em função dos custos gerados pelas leis trabalhistas, como impostos sobre a folha de pagamento, seguro desemprego e intensidade da fiscalização governamental sobre o setor informal da economia. Outras questões que associam o desemprego a aspectos institucionais podem ser encontradas em Fernandes \& Chahad (2002), Fernandes (1998) e Fernandes \& Cacciamali (1993).

O reflexo das regulamentações que tornam o mercado de trabalho menos flexível incide diretamente sobre rendimento, distribuição espacial dos trabalhadores e nível de emprego devido aos custos diretos e indiretos envolvidos na demissão e contratação de trabalhadores, como multas e encargos trabalhistas ou treinamento de pessoal. Circunscrita ao interesse desse estudo está a avaliação do grau de persistência na série de desemprego na maior região metropolitana brasileira, como uma possível interpretação da rigidez nesse mercado de trabalho. Blanchard \& Summers (1986) foram os primeiros a expor evidências de que a persistência desempenha um importante caráter na análise do desemprego. Os autores investigaram o desemprego na Europa na década de 1980 e avaliaram que os choques sobre as taxas de desemprego possuem efeitos mais persistentes do que as teorias tradicionais podem explicar, produzindo significativas implicações em termos de políticas públicas de emprego. Esse comportamento foi denominado pelos autores como "histerese", em referência ao fenômeno oriundo da Física. De acordo com Smith (1994) o fenômeno da histerese é resultante de uma elevada correlação (positiva) entre o desemprego passado e presente. A histerese na taxa de desemprego, seguindo o conceito físico em stritu sensu, implica a existência de uma tendência estocástica na série histórica da variável (Ball 2002). Como consequência, a estrutura de memória da série torna-se influenciada permanentemente pelos choques passados que incidiram sobre a série. A hipótese da histerese contrapõe-se, portanto, à concepção de Friedman (1968) de existência de uma taxa natural de desemprego, também chamada de doutrina NAIRU.

Diversas pesquisas na área de economia aplicada procuraram avaliar a hipótese da histerese no desemprego por meio da aplicação de testes de raízes unitárias. Song \& Wu (1998), por exemplo, analisaram o referido fenômeno 
no desemprego dos E.U.A. utilizando dados anuais desagregados de desemprego de quarenta e oito estados norte-americanos. Os resultados apontados pelos autores indicam a existência de uma tendência estocástica nas séries, em consonância com a hipótese de histerese. Clement et al. (2005) revisitaram o estudo de Song \& Wu (op. cit.) utilizando dados mensais desagregados por estados e regiões. Os autores afirmam ter encontrado indícios contrários à pesquisa anterior, embora dezessete entre as sessenta e cinco séries analisadas no estudo não tenham rejeitado a hipótese de raiz unitária e que outras dezessete séries somente rejeitaram essa hipótese ao nível de $10 \%$ de significância. Camarero \& Tamarit (2004) testaram a hipótese da histerese na taxa de desemprego dos dezenove países-membros da Organização para a Cooperação e Desenvolvimento Econômico (OCDE). Foi utilizado um modelo de teste de raiz unitária para dados em painel, capaz de captar a interdependência dos dados entre os países. Em suas conclusões, foi relatado que, ao contrário dos testes aplicados aos países individualmente, o teste multivariado para dados em painel revelou um alto índice de rejeição da hipótese nula de não estacionariedade, contrariando, portanto, a hipótese testada. Em Gomes \& Gomes da Silva (2009) os autores procuraram verificar a validade empírica das doutrinas NAIRU e Histerese sobre o comportamento do desemprego no Brasil com base nos testes de raízes unitárias com quebras estruturais. Os resultados da pesquisa indicam que a hipótese da histerese somente não é corroborada na região metropolitana do Rio de Janeiro. Adicionalmente, o estudo buscou retratar a existência de uma convergência estocástica entre as taxas de desemprego das regiões que não rejeitaram a hipótese de histerese. Nesse caso, apenas a região de Porto Alegre revelou indícios contrários à convergência.

Sob o escopo dos modelos de integração fracionária, no entanto, a histerese na taxa de desemprego pode ganhar um novo caráter: o caráter estatístico conhecido como memória longa. Os modelos de memória longa revelam que os efeitos dos choques em uma série temporal, embora influenciem fortemente seu futuro, se dissipam em longos intervalos de tempo. Nesse caso, os choques passados não permanecem definitivamente na estrutura de memória da série como no caso das tendências estocásticas, mas apenas temporariamente, durante décadas, por exemplo.

A evolução dos modelos de memória longa vem permitindo a identificação e a diferenciação mais precisa dos comportamentos permanente e persistente em séries temporais. Granger \& Joyeux (1980) expuseram que a modelagem econométrica baseada na integração unitária desconsidera importantes informações sobre a dinâmica das séries temporais contida nas bandas de frequências próximas a zero. Entretanto, como expresso em Granger (1966), a maioria das variáveis (macro)econômicas apresenta concentração de energia espectral nessa faixa de frequência. A análise de memória longa possui vantagens significativas no estudo da dinâmica do ajustamento no médio e longo prazos em comparação à análise tradicional de raiz unitária, pois ela consegue captar adequadamente as informações contidas nas baixas frequências das séries temporais por meio do conceito matemático da integração/diferenciação fracionária.

Os modelos de integração unitária e os respectivos testes de raiz unitária baseiam-se na dicotomia " $H_{0}$ : séries com uma tendência estocástica" vs. " $H_{1}$ : séries fracamente estacionárias", denominada como paradigma econométrico $I(1)-I(0)$. Esse paradigma fundamenta, por exemplo, os modelos autoregressivos integrados de médias móveis (ARIMA) utilizados por Box \& Jen- 
kins (1976) e os modelos de análise de co-integração de Engle \& Granger (1987) e de Johansen (1995). Diebold \& Rudebush (1991), no entanto, realizaram experimentos de Monte Carlo envolvendo processos com memória longa e perceberam o baixo poder do teste de raiz unitária $D F$ de Dickey \& Fuller (1979) em distinguir os casos de raiz unitária e de estacionariedade quando as séries são fracionariamente integradas. Em outro trabalho, Hassler \& Wolters (1994) realizaram experimentos semelhantes, porém utilizando a versão "aumentada" do teste DF proposta por Said \& Dickey (1984) e o teste PP de Phillips \& Perron (1988). Os autores perceberam potência dos testes $D F$ e $P P$ somente no intervalo de estacionariedade assintótica, ou seja, quando a ordem de integração $d$ da série é tal que $-1 / 2<d<1 / 2$. Os autores mostraram a inferioridade do teste $A D F$, que sofre influências negativas em relação ao aumento do número de defasagens utilizadas. No intervalo de não estacionariedade, mas com reversibilidade à média, $1 / 2 \leq d<1$, os testes de raiz unitária mostram baixa potência em rejeitar a hipótese $H_{0}: d=1$ contra a hipótese $H_{1}: d<1$. Lee (1996) abordaram o comportamento do teste de estacionariedade KPSS proposto por Kwiatkowski et al. (1992) em séries com memória longa. Seus resultados mostram a baixa potência do teste em identificar $H_{0}: d=0$ vs. $H_{1}: d>0$, quando $d$ é fracionário.

O baixo poder dos testes de raízes unitárias implica a baixa capacidade desses modelos em averiguar o comportamento persistente e, portanto, a hipótese de histerese no desemprego. Implica, também, que a modelagem baseada nos modelos ARIMA tende a superestimar os efeitos dos choques imprimindo-os um caráter permanente. Os efeitos dos choques sobre modelos com integração no intervalo $0<d<1$ tendem a se dissipar ao longo do tempo, enquanto que em séries integradas com $d \geq 1$ eles são incorporados permanentemente. Os modelos de longa dependência surgem, portanto, como uma resposta a essa lacuna.

Diebold \& Rudebush (1989) realizaram um dos primeiros trabalhos de macroeconometria aplicada sob o arcabouço da integração fracionária considerando o tema da persistência no produto e no desemprego norte-americanos. Os autores utilizaram os modelos auto-regressivos fracionariamente integrados de médias móveis (ARFIMA) para modelar as séries e calcular suas funções impulso-resposta acumuladas. Os resultados encontrados na pesquisa apontam um comportamento de reversibilidade à média no longo prazo no produto e na taxa de desemprego, contrários aos resultados apontados por Campbell (1987).

Estudos econômicos aplicados envolvendo dados brasileiros têm mostrado que diferentes séries nacionais apresentam padrões de memória longa. Fava \& Alves (1998) e Reisen et al. (2003), por exemplo, analisaram séries de inflação com base nos modelos ARFIMA. Esses estudos utilizaram diferentes estimadores do parâmetro de integração fracionária e seus resultados revelam a existência de importantes componentes de longa dependência nas séries, não captados pela abordagem tradicional. Gomes \& Gomes da Silva (2009) examinaram a questão da persistência na taxa de desemprego da região metropolitana de São Paulo em diferentes níveis de desagregação por gênero do indivíduo, etnia e sua posição no núcleo familiar. Os autores utilizaram os modelos de raízes unitárias com quebras estruturais e os modelos ARFIMA sobre séries ajustadas sazonalmente. Suas conclusões indicam que não se pode rejeitar a hipótese de existência de uma raiz unitária nas séries de desemprego, mesmo considerando possíveis quebras estruturais. 
Seguindo a linha de pesquisa que analisa a persistência por meio dos modelos de integração fracionária, esse trabalho tem como objetivo identificar o grau de persistência em uma das principais séries de desemprego da região metropolitana de São Paulo, a Pesquisa de Emprego e Desemprego (PED), calculada pelos institutos SEADE e DIEESE. As séries brasileiras sobre desemprego, no entanto, são particularmente marcadas por fortes influências sazonais que dificultam as avaliações estatísticas sobre a existência de grandes ciclos ou de movimentos convergentes a uma taxa natural de desemprego. Por esse motivo, adotou-se um modelo que possibilita a investigar a ocorrência simultânea de componentes de memória longa sazonal e não sazonal em uma série temporal: o modelo auto-regressivo fracionariamente integrado sazonal e com médias móveis (SARFIMA). ${ }^{1}$ Ooms (1995) expõe na forma de um compêndio o desenvolvimento da pesquisa sobre a memória longa sazonal.

Pesquisas em diferentes áreas têm se apoiado em modelos de integração fracionária para avaliar as persistências sazonal e cíclica principalmente em fenômenos naturais. Na Economia, Gil-Alana (2007) examinou os dados macroeconômicos norte-americanos utilizados por Nelson \& Plosser (1982) para fundamentar sua importante crítica econométrica. O autor analisou a presença dos padrões de memória longa em observações consecutivas conjuntamente à memória longa em movimentos ć́clicos. A série anual da taxa de desemprego nos E.U.A. apresentou um comportamento de memória longa, embora não estacionário, comparativamente inferior ao das outras séries macroeconômicas estudadas, enquanto que seu nível de persistência cíclica mostrouse superior. O mesmo autor examinou em Gil-Alana (2008) a integração e co-integração fracionárias sazonal na série de demanda por trabalho dinamarquesa e relatou a influência da subespecificação do modelo nos resultados caso o componente de memória longa sazonal não seja considerado. Outras importantes referências bibliográficas sobre o tema são mencionadas oportunamente ao logo do trabalho.

A análise de memória longa será o instrumento utilizado para duas finalidades nesse estudo: extrair as estruturas de memória longa sazonal e não sazonal da série da $P E D$ e avaliar o grau de persistência dos choques gerado pela união dos efeitos dos dois componentes. ${ }^{2}$ Adicionalmente, este estudo confronta os resultados relativos à persistência na série obtidos com as modelagens $I(1)-I(0)$ e fracionária. Em resumo, os resultados encontrados corroboram a hipótese de histerese na taxa de desemprego da região metropolitana de São Paulo. O uso do instrumental de integração fracionária sazonal e não sazonal, diferentemente de outros trabalhos, permitiu identificar que o fenômeno percebido outrora é resultado da conjunção das estruturas de memória sazonal e não sazonal presentes na série de desemprego.

O conjunto dessas avaliações está organizado em seções dispostas da seguinte forma: na seção 2, são introduzidos os principais conceitos relativos aos modelos de integração fracionária sazonal e suas propriedades; a seção 3 expõe os dados utilizados no trabalho e confronta os resultados obtidos com a metodologia sazonal tradicional com os do modelo de memória longa sazonal; a seção 4 reúne as principais conclusões do estudo.

\footnotetext{
${ }^{1}$ Tradução de seasonal auto-regressive fractionally integrated moving-average model.

${ }^{2} \mathrm{O}$ termo persistência é utilizado na literatura econométrica como uma referência à duração dos efeitos dos choques incidentes em uma série temporal.
} 


\section{Persistência em Modelos de Memória Longa Sazonal}

A teoria da integração fracionária permite generalizações dos modelos de memória longa a fim de adequá-los às condições observadas de elevada persistência sazonal com diferentes periodicidades. Esses modelos são chamados de "modelos de memória longa sazonal". Hosking (1981) sugeriu resumidamente um modelo de memória longa capaz de captar componentes de memória longa associados às diferentes frequências espectrais:

$$
\left(1-2 \vartheta B+B^{2}\right)^{\delta} y_{t}=\varepsilon_{t},
$$

em que $B$ é o operador diferença, $B^{i} x_{t}=x_{t-i} ; \vartheta=\cos (2 \pi v)$, $\delta$ é o parâmetro de integração, tal que $\delta \in \mathbb{R}$, e $\varepsilon_{t}$ é um processo ruído branco com variância $\sigma_{\varepsilon}^{2}$. $O$ processo possui função de densidade espectral (FDE) dada por:

$$
f_{y}(v)=\sigma_{\varepsilon}^{2}[2|\cos (2 \pi v)-\vartheta|]^{-2 \delta},-1 / 2<v<1 / 2
$$

O processo (1) foi explorado por Andel (1986), Gray et al. (1989), Chung (1996), Giraitis \& Leipus (1995), Woodward et al. (1998) e Hsu \& Tsai (2009). Particularmente na frequência zero, tem-se $\vartheta_{i}=0$ e (1) torna-se um processo com memória longa tradicional com ordem de integração fracionária $d=2 \delta$. De acordo com Gray et al. (1989), o processo (1) é estacionário se $|\vartheta|=1$ e $-1 / 4<\delta<1 / 4$ ou se $|\vartheta|<1$ e $-1 / 2<\delta<1 / 2$. Os autores também mostraram que, sob a condição de estacionariedade, o processo pode ser expresso na forma de um polinômio de Gegenbauer, fonte de sua denominação mais difundida. ${ }^{3} \mathrm{Na}$ frequência $v_{G}$, denominada frequência de Gegenbauer, a FDE apresenta uma singularidade ou pólo.

Giraitis \& Leipus (op. cit.) e Woodward et al (op. cit.) propuseram versões multifatoriais do processo de Gegenbauer, denominados, respectivamente, por modelo ARUMA e GARMA k-fator. O modelo ARUMA $\left(0, \delta_{0}, \delta_{i}, \ldots, \delta_{s / 2}, 0\right)$ possui representação:

$$
\prod_{j=0}^{s / 2}\left[\left(1-2 \vartheta_{j} B+B^{2}\right)\right]^{\delta_{j}} y_{t}=\varepsilon_{t},
$$

com $\vartheta_{j}=\cos \left(v_{j}\right), v_{j}=\frac{2 \pi j}{s}, j=0,1, \ldots,[s / 2]$ e $\delta_{0}=\left(d+d_{s}\right) / 2$ para frequência zero, $v_{0}=0$ e $\delta_{i}=d_{s}, i=1,2, \ldots,(s / 2)-1$, para as frequências sazonais em $0<$ $v<\pi / s$ e $\delta_{[s / 2]}=d_{s} / 2$, para $v_{s / 2}=\pi$, tal que $\left(1-B^{s}\right)^{d_{s}}=\nabla^{d_{s}}$. A sequência $\left\{\varepsilon_{t}\right\}_{\in \mathbb{Z}}$ é um processo ruído branco gaussiano. $\mathrm{O}$ modelo sugerido por Woodward $e t$ al (op. cit.),

$$
\prod_{i=1}^{k}\left[\left(1-2 \vartheta_{i} B+B^{2}\right)\right]^{\delta_{i}} y_{t}=\varepsilon_{t}
$$

cujo espectro

$$
f_{y}(v)=\sigma_{\varepsilon}^{2} \prod_{i=1}^{k}[2|\cos (2 \pi v)-\vartheta|]^{-2 \delta_{i}},-1 / 2<v<1 / 2,
$$

\footnotetext{
${ }^{3} \mathrm{~A}$ versão do processo que inclui termos auto-regressivos e de médias móveis é denominada Gegenbauer-ARMA (GARMA).
} 
compreende $k$ singularidades nas frequências $v_{G_{i}}=\left(\cos ^{-1} \vartheta_{i}\right) / 2 \pi, i=1, \ldots, k$, associadas aos componentes de memória longa.

Reisen et al. (2006) mostraram que o filtro $\nabla^{d, d_{s}}=(1-B)^{d}\left(1-B^{s}\right)^{d_{s}},\left(d, d_{s}\right) \in$ $\mathbb{R}$, pode ser fatorado em diferentes termos e frequências por meio da expansão:

$$
\begin{gathered}
\nabla^{d, d_{s}}=\prod_{j=0}^{s / 2}\left[\left(1-B e^{i v_{j}}\right)\left(1-B e^{-i v_{j}}\right)\right]^{\delta_{j}} \\
=\prod_{j=0}^{s / 2}\left[\left(1-2 \vartheta_{j} B+B^{2}\right)\right]^{\delta_{j}}, \\
v_{j}=\frac{2 \pi j}{s}, \vartheta_{j}=\cos \left(v_{j}\right), j=0,1, \ldots, s / 2 .
\end{gathered}
$$

Como resultado, os autores provaram que o modelo $\operatorname{SARFIMA}(0 ; d ; 0) \times\left(0 ; d_{s} ; 0\right)$ é um caso particular do modelo $\operatorname{ARUMA}\left(0, \delta_{0}, \delta_{i}, \ldots, \delta_{s / 2}, 0\right)$. Porter-Hudak 1990 (1990), Hassler \& Wolters (1994), Arteche \& Robinson (2000), Arteche (2003), Reisen et al. (2006), Palma (2007), Diongue et al. (2008) e Bisognin \& Lopes (2009) são importantes referências sobre o modelo SARFIMA ou AFIRSMA. ${ }^{4}$ Reisen et al (op. cit.) compararam a eficiência de "versões sazonais" do estimador semiparamétrico GPH de Geweke \& Porter-Hudak (1983) na estimação do parâmetro de integração fracionária sazonal $d_{s}$. Essas pesquisas também avaliaram o estimador de máxima verossimilhança gaussiano $(M V)$, descrito em Beran (1994) para a estimação de $d_{s}$. Os resultados dos autores revelam a superioriedade do estimador $M V$ em comparação às versões sazonais do $G P H$. Seguindo essa indicação, será utilizado na análise aplicada desse estudo o estimador de $M V$ calculado com base na aproximação de Whittle no domínio da frequência, que permite uma redução significativa no tempo de cálculo.

O modelo $\operatorname{SARFIMA}(0 ; d ; 0) \times\left(0 ; d_{s} ; 0\right)$, que compreende um componente de memória longa sazonal e um de memória longa não sazonal pode ser definido, com base em Porter-Hudak (op. cit.) e Bisognin \& Lopes (2007), da seguinte forma:

Definição 1. O processo estocástico $\left\{s_{t}\right\}_{t \in \mathbb{Z}}$ é um modelo $\operatorname{SARFIMA}(p ; d ; q) \times$ $\left(P ; d_{s} ; Q\right)_{s}$ se representar uma solução da equação em diferenças na forma:

$$
\phi(B) \Phi\left(B^{s}\right)(1-B)^{d}(1-B)^{d_{s}}\left(s_{t}-\mu\right)=\theta(B) \Theta\left(B^{s}\right) \varepsilon_{t},
$$

em que $\left\{\varepsilon_{t}\right\}_{t \in \mathbb{Z}}$ é um processo ruído branco, $\mu$ é a média do processo, $B^{j}$ é o operador defasagem, ou seja, $B^{j} s_{t}=s_{t-j}$. Os termos $(1-B)^{d}$ e $(1-B)^{d_{s}}$ são, respectivamente, os operadores diferença fracionária e diferença fracionária sazonal, com ordens de integração d e $d_{s}$. Os polinômios $\phi(B)=1-\phi_{1} B^{1}-$ $\cdots-\phi_{p} B^{p}, \Phi\left(B^{s}\right)=1-\Phi_{1} B^{1 s}-\cdots-\Phi_{P} B^{P s}, \theta(B)=1-\theta_{1} B^{1}-\cdots-\theta_{q} B^{q}$ e $\Theta\left(B^{s}\right)=$ $1-\Theta_{1} B^{1 s}-\cdots-\Theta_{Q} B^{Q s}$ são, respectivamente, os polinômios auto-regressivos, auto-regressivos sazonais, de médias móveis e de médias móveis sazonais de ordens $p, q, P$ e $Q$, cujas raízes características de $\phi(B)=0, \Phi\left(B^{S}\right)=0, \theta(B)=0$ e $\Theta\left(B^{s}\right)=0$ caem todas fora do circulo unitário.

\footnotetext{
${ }^{4}$ Adicionalmente é encontrada a denominação FARISMA para o mesmo modelo.
} 
O operador defasagem (sazonal), $B^{s}$, atua sobre as defasagens múltiplas de $s, B^{j s} s_{t}=s_{t-j s}$. O operador diferença fracionária $\Delta^{d}=(1-B)^{d}$ aplica o filtro:

$$
(1-B)^{d}=\sum_{k=0}^{+\infty} \frac{\Gamma(-d+k)}{\Gamma(-d) \Gamma(k+1)} B^{k}
$$

em defasagens consecutivas, enquanto o operador diferença fracionária sazonal $\Delta^{d_{s}}=(1-B)^{d_{s}}$ aplica o filtro para as defasagens sazonais e zero para os demais. O operador diferença fracionária expresso em (8) apóia-se na função gama, $\Gamma(u)=\int_{0}^{+\infty} e^{-t} t^{u-1} d t$, que interpola uma expansão binomial.

De acordo com o Teorema 2.2 de Bisognin \& Lopes (2009) o processo (7) será estacionário e invertível se $d+d_{s}<1 / 2$, para $d_{s}<1 / 2$ e possuirá $F D E$ dada por:

$$
f_{y}(v)=\frac{\sigma_{\varepsilon}^{2}}{2 \pi}\left|1-e^{-i v}\right|^{-2 d}\left|1-e^{-i s v}\right|^{-2 d_{s}} \frac{\left|\theta\left(e^{-i v}\right)\right|^{2}\left|\Theta\left(e^{-i s v}\right)\right|^{2}}{\left|\phi\left(e^{-i v}\right)\right|^{2}\left|\Phi\left(e^{-i s v}\right)\right|^{2}},
$$

onde $0<v \leq \pi, e^{i \alpha}=\cos (\alpha)+i \sin (\alpha), i \in \mathbb{C}$, é a exponencial complexa denominada por equação de Euller. O modelo SARFIMA exibe múltiplas singularidades espectrais em sua $F D E$ nas frequências sazonais $v_{j}=2 \pi j / s, j=1,2, \ldots, s / 2$. A Figura 1 mostra exemplos da FDE dos modelos SARFIMA para periodicidades anuais e semestrais nas frequências $0<v<\pi$.
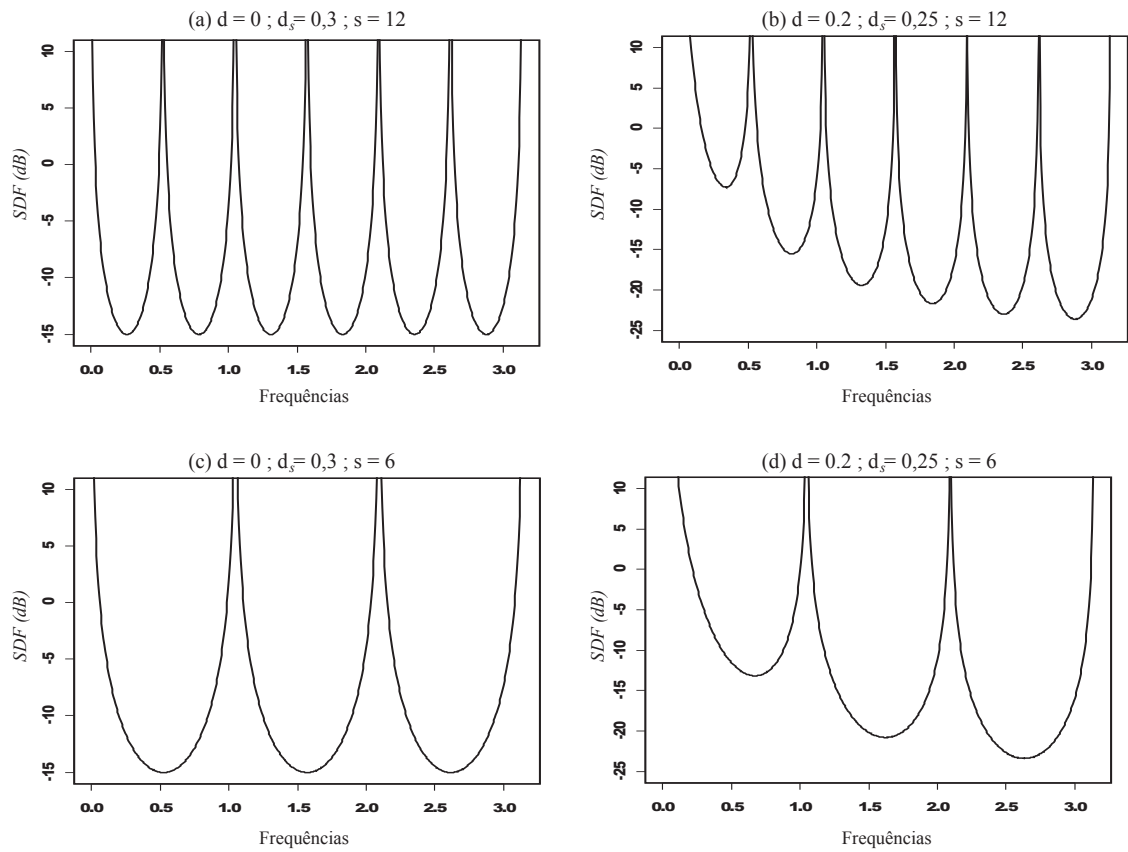

a) $\operatorname{SARFIMA}(0,0,0) \times(0,0.3,0)_{12}$

b) $\operatorname{SARFIMA}(0,0.2,0) \times(0,0.25,0)_{12}$

c) $\operatorname{SARFIMA}(0,0.0,0) \times(0,0.3,0)_{6}$

d) $\operatorname{SARFIMA}(0,0.2,0) \times(0,0.25,0)_{6}$

Figura 1: Função de desnsidade espectral teórica dos modelos SARFIMA

Baseando-se na especificação do modelo SARFIMA, pode-se calcular a função impulso-resposta que mede os efeitos dos choques sobre a dinâmica da série: $\frac{\partial s_{t+k}}{\partial \varepsilon_{t}}=\psi_{k}, \operatorname{com} k=0,1,2$, dots e $\psi_{0}=1$. Assumindo $\mu=0$ em (7): 


$$
s_{t}=(1-B)^{-d}(1-B)^{-d_{s}} \frac{\theta(B)}{\phi(B)} \frac{\Theta\left(B^{s}\right)}{\Phi\left(B^{s}\right)} \varepsilon_{t}
$$

$\mathrm{ou}$

$$
s_{t}=\psi^{s}(\tilde{B}) \varepsilon_{t}, \quad \operatorname{com} \psi^{s}(\tilde{B})=(1-B)^{-d}\left(1-B^{s}\right)^{-d_{s}} \frac{\theta(B)}{\phi(B)} \frac{\Theta\left(B^{s}\right)}{\Phi\left(B^{s}\right)} .
$$

O polinômio

$$
\psi^{S}(\tilde{B})=1+\psi_{1} \tilde{B}^{1}+\psi_{2} \tilde{B}^{2}+\ldots
$$

envolve operadores defasagens temporais $\tilde{B}$ que sobrepõem os efeitos da memória longa e curta em autocorrelações consecutivas e sazonais. Os coeficientes $\psi_{k}^{s}$ com $k=0,1,2$, dots e $\psi_{0}^{s}=1$ de (12) extraídos a partir de (11) definem a função impulso-resposta sazonal $(F I R-S)$ do modelo $\operatorname{SARFIMA}(p ; d ; q) \times$ $\left(P ; d_{s} ; Q\right)_{s}$.

\section{Persistência na Série da Pesquisa de Emprego e Desemprego da Região Metropolitana de São Paulo}

A série abordada nesse estudo é composta por observações mensais do indicador de desemprego na Região Metropolitana de São Paulo (RMSP), calculado em conjunto pelos institutos SEADE e DIEESE. ${ }^{5}$ Na Figura 2, são mostrados dois gráficos referentes à $P E D$.

(a) PED (RMSP) - Série no Nível

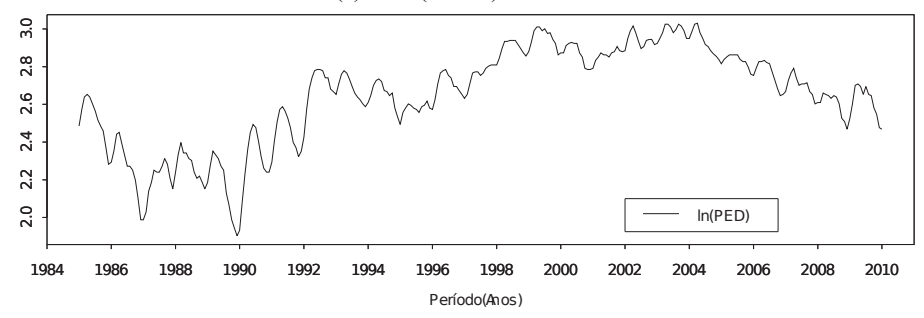

(b) PED (RMSP) - Séries Sem Tendência Linear

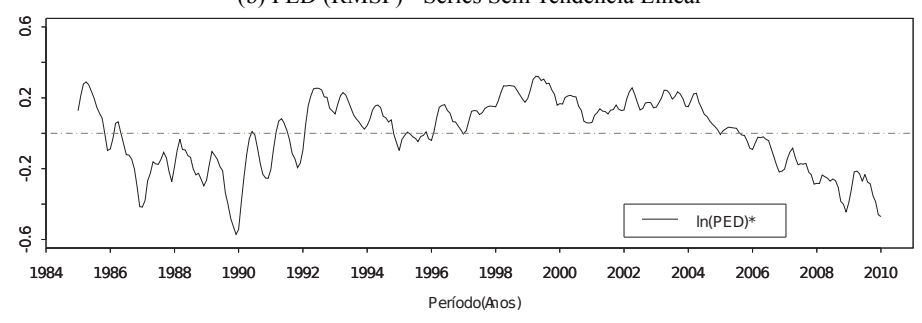

Fonte: SEADE/DIEESE

Figura 2: Taxa de desemprego(PED) - Região Metropolitana de São Paulo (RMSP)

No primeiro gráfico, a série é mostrada em seu nível histórico, e no segundo, é exibida sem a sua tendência linear e intercepto, ambos extraídos por meio do método de mínimos quadrados. A série representa o percentual de

\footnotetext{
${ }^{5}$ Compreende o desemprego aberto e o desemprego oculto.
} 
desempregados na Região Metropolitana de São Paulo e compõe 301 observações, com início em janeiro de 1985 e estendendo-se até janeiro de 2010. Visualmente, o gráfico da série sem tendência linear induz à suposição de um comportamento de reversibilidade à média, ou seja, de que a PED gravita estocasticamente em torno de um nível médio. Esse padrão é natural aos processos estocásticos não estacionários e integrados fracionariamente no intervalo $1 / 2<d<1$. As funções de autocorrelação $(F A C)$ e de autocorrelação parcial $(F A C-P)$ da $P E D$ são mostradas na Figura 3.

(a) Função de Autocorrelação

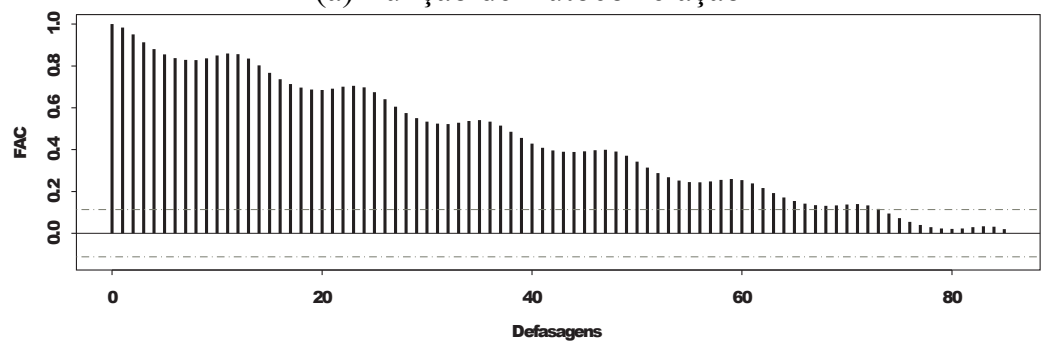

(b) Função de Autocorrelação Parcial

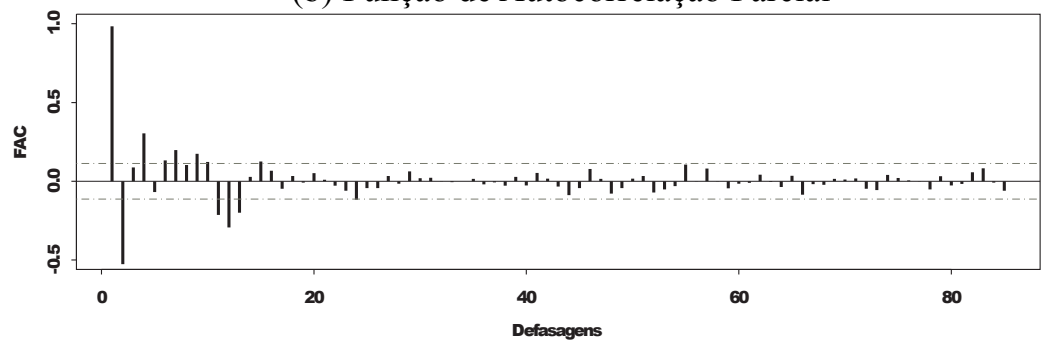

Figura 3: $F A C$ e $F A C-P$ da série $P E D$

No gráfico da $F A C$, percebe-se um decaimento hiperbólico "ondular" da função, apresentando valores estatisticamente diferentes de zero até aproximadamente a defasagem 70. A silhueta lentamente decrescente da FAC é indicativa da presença de memória longa na série, enquanto seu movimento na forma senoidal reflete um padrão tipicamente sazonal. A $F A C-P$, na qual são excluídas as influências de defasagens intermediárias, indica a existência de autocorrelações de curto prazo na série. A Figura 4 mostra a FDE estimada da série da $P E D$ na escala decibel $(d B)$. Além da concentração de energia espectral nas frequências mais baixas, o estimador espectral periodograma exibe dois picos nas frequências 0.08148 e 0.1666 referentes, respectivamente, a períodos de aproximadamente 12 e 6 meses, indicando a possível presença de dois componentes sazonais na série.

Inicialmente são considerados alguns aspectos da abordagem $I(1)-I(0)$. Foram aplicados os testes de raiz unitária ADF, DF-GLS, ERS, MEPP e KPSS sobre a série. De acordo com os resultados da Tabela 1, nenhum dos testes rejeitou a hipótese da série ser $I(1)$, independentemente dos termos deterministas incluídos em suas formas funcionais. Este resultado indica a existência de uma tendência estocástica na série. ${ }^{6}$

\footnotetext{
${ }^{6}$ Foi avaliada também a existência de três e duas raízes unitárias na série, por meio do teste de Dickey \& Pantula (1987). O teste rejeitou a hipótese de múltiplas raízes unitárias ao nível de
} 


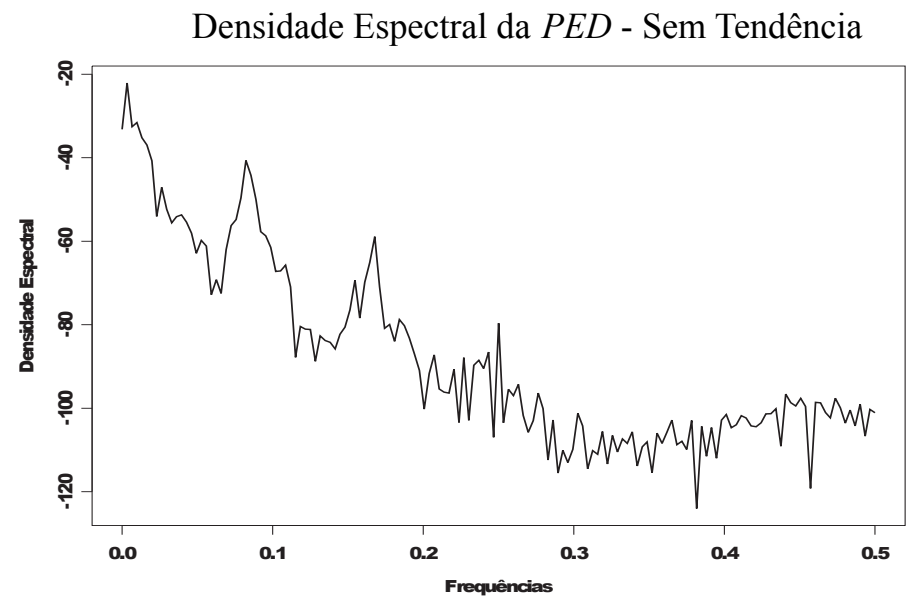

Figura 4: Espectro da $P E D$ - baseado na série sem tendência linear

Tabela 1: Teste de raiz unitária

\begin{tabular}{clcccc}
\hline \multirow{2}{*}{ Variáveis } & \multicolumn{3}{c}{ Termos Deterministas } & \\
\cline { 2 - 4 } & $\begin{array}{c}\text { Constante e } \\
\text { Tendência }\end{array}$ & Constante & $\begin{array}{c}\text { Sem Const. } \\
\text { e Tend. }\end{array}$ & Decisão \\
\hline ADF & $-1,6549$ & $-1,8816$ & 0,1156 & $I(1)$ \\
DED & DF-GLS & $-1,9527$ & $-1,4812$ & 0,1156 & $I(1)$ \\
& $E R S$ & 9,8470 & 5,4656 & 6,4667 & $I(1)$ \\
& $M E P P$ & $-2,0841$ & $-1,5172$ & 1,2344 & $I(1)$ \\
KPSS & $0,7374^{* *}$ & $2,9244^{* *}$ & & $I(1)$ \\
\hline
\end{tabular}

Testes ADF, DF-GLS, ERS e MEPP: $H_{0}: 1$ Raiz Unitária ; Teste KPSS: $H_{0}$ :

Série Estacionária

${ }^{*}$ Rejeita $H_{0}$ ao Nível de 5\% de Significância.

${ }^{* *}$ Rejeita $H_{0}$ ao Nível de $1 \%$ de Significância.

Na Figura 5, são mostrados os gráficos e as $F A C$ da $P E D$ em primeiras diferenças e em primeiras diferenças sazonais de 12 meses. Pelo gráfico (b), referente à $F A C$ da primeira diferença da série, nota-se um componente sazonal extremamente persistente. O gráfico (d) exibe a $F A C$ da série em primeiras diferenças consecutivas e sazonais de 12 meses.

Notam-se no gráfico (d), no entanto, sinais de que a série pode ter sido "sazonalmente superdiferenciada". Embora a diferença $\Delta^{12}=\left(1-B^{12}\right)$ tenha eliminado grande parte das longas correlações periódicas, o surgimento de elevadas autocorrelações negativas em torno da defasagem 12 , não presentes na FAC do gráfico (b), são evidências do "superdimensionamento" do componente sazonal. Como resultado, a modelagem tradicional da série filtrada mostrada no gráfico (c), pela metodologia $A R M A(p ; q)$, necessitaria suprir o modelo com termos capazes de expurgar as autocorrelações introduzidas artificialmente pela superdiferenciação. A Tabela 2 destaca quatro modelos $\operatorname{SARIMA}(p ; d ; q) \times\left(P ; d_{s} ; Q\right)_{s}$ ajustados à série da $P E D$ pelo procedimento de máxima verossimilhança. ${ }^{7} \mathrm{O}$ modelo que se mostrou mais adequado foi $\mathrm{o}$

$1 \%$ de significância: $\hat{\tau}_{3}=-7,17$ e $\hat{\tau}_{2}=-4,19$.

${ }^{7}$ Além das especificações exibidas, outras formas auto-regressivas e de médias móveis de 
(a) $\mathrm{D}(P E D)$

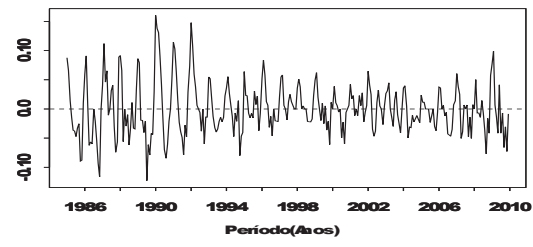

(c) $\mathrm{D}(\mathrm{D}(P E D), 12)$

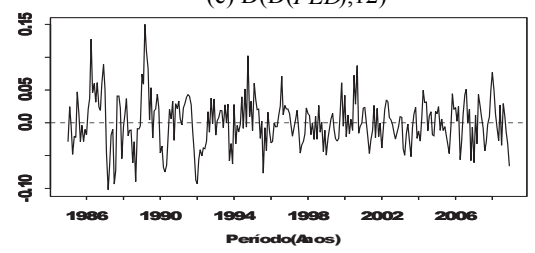

(b) $F A C$ da $\mathrm{D}(P E D)$

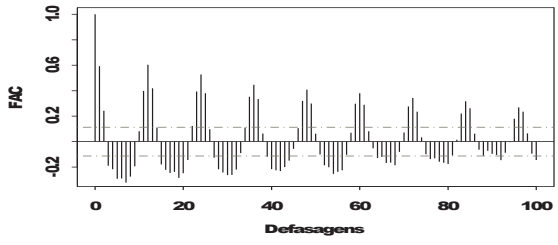

(d) FAC da $\mathrm{D}(\mathrm{D}(P E D), 12)$

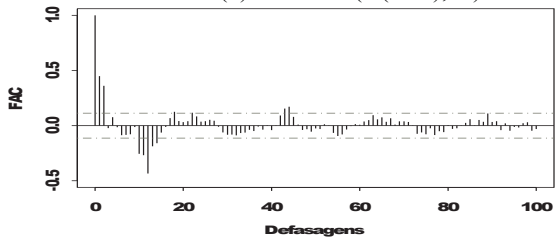

Figura 5: Gráficos da série $P E D$ em diferenças consecutivas e sazonais unitárias e de suas respectivas $F A C$

$\operatorname{SARIMA}(6 ; 1 ; 0) \times(1 ; 0 ; 1)_{12}$, apresentado nas duas últimas colunas da tabela. Essa especificação conseguiu capturar as autocorrelações consecutivas e sazonais de maneira satisfatória.

No escopo dos modelos de memória longa sazonal, a série $P E D$ foi analisada com base no modelo $\operatorname{SARFIMA}(p ; d ; q) \times\left(P ; d_{s} ; Q\right)_{s}$. A Tabela 3 mostra os modelos estimados que produziram os melhores resultados de acordo com o critério de significância dos parâmetros, critério de informação bayesiana (BIC), parcimônia e inexistência de autocorrelações residuais. Além dos modelos expostos, foram avaliadas especificações envolvendo termos sazonais, $S A R(P)_{s}$ e $S M A(P)_{s}$. Nenhuma delas, no entanto, gerou resultados superiores. $\mathrm{O}$ modelo escolhido como aquele que representa melhor a dinâmica da série PED foi o SARFIMA $(6 ; 0,9156 ; 0) \times(0 ; 0,3642 ; 0)_{12}$, apesar de exibir um coeficiente $A R(5)$ estatisticamente nulo ao nível de significância de $8 \%$. Optou-se por esse modelo pela necessidade de captação de autocorrelações residuais de curto prazo presentes na série. Nos demais modelos, uma autocorrelação de ordem 6, provavelmente aquela captada pelo espectro na frequência 0,1666 , persistia sistematicamente na $F A C$. A inclusão de um termo auto-regressivo de mesma ordem expurgou seu efeito, como pode ser percebido na Figura 6.

$\mathrm{O}$ espectro da série filtrada pelo $\operatorname{SARFIMA}(6 ; 0,9156 ; 0) \times(0 ; 0,3642 ; 0)_{12}$ revela-se "aplainado" e, portanto, entende-se que a energia espectral concentrada da segunda singularidade percebida no periodograma da série original não é suficientemente alta para se refletir em um padrão sazonal com período de 6 meses. $^{8}$ O modelo de memória longa indica a existência de um componente de memória longa sazonal na $P E D$, assintoticamente estacionário, com período de 12 meses e um componente de memória longa relativo às autocorrelações sucessivas, não estacionário e reversível à média.

A persistência da série relativa às inovações foi avaliada por meio da função impulso-resposta, modificada para compreender tanto a componente de memória longa sazonal quanto a não sazonal, além da estrutura de memória

ordens mais elevadas foram estimadas, porém nenhuma produziu resultados relevantes.

${ }^{8}$ Adicionalmente, foi realizado o teste de Whittle para avaliar a significância da periodicidade nessa frequência. O resultado rejeitou, ao nível de $5 \%$ de significância, a hipótese de existência da sazonalidade de 6 meses. 


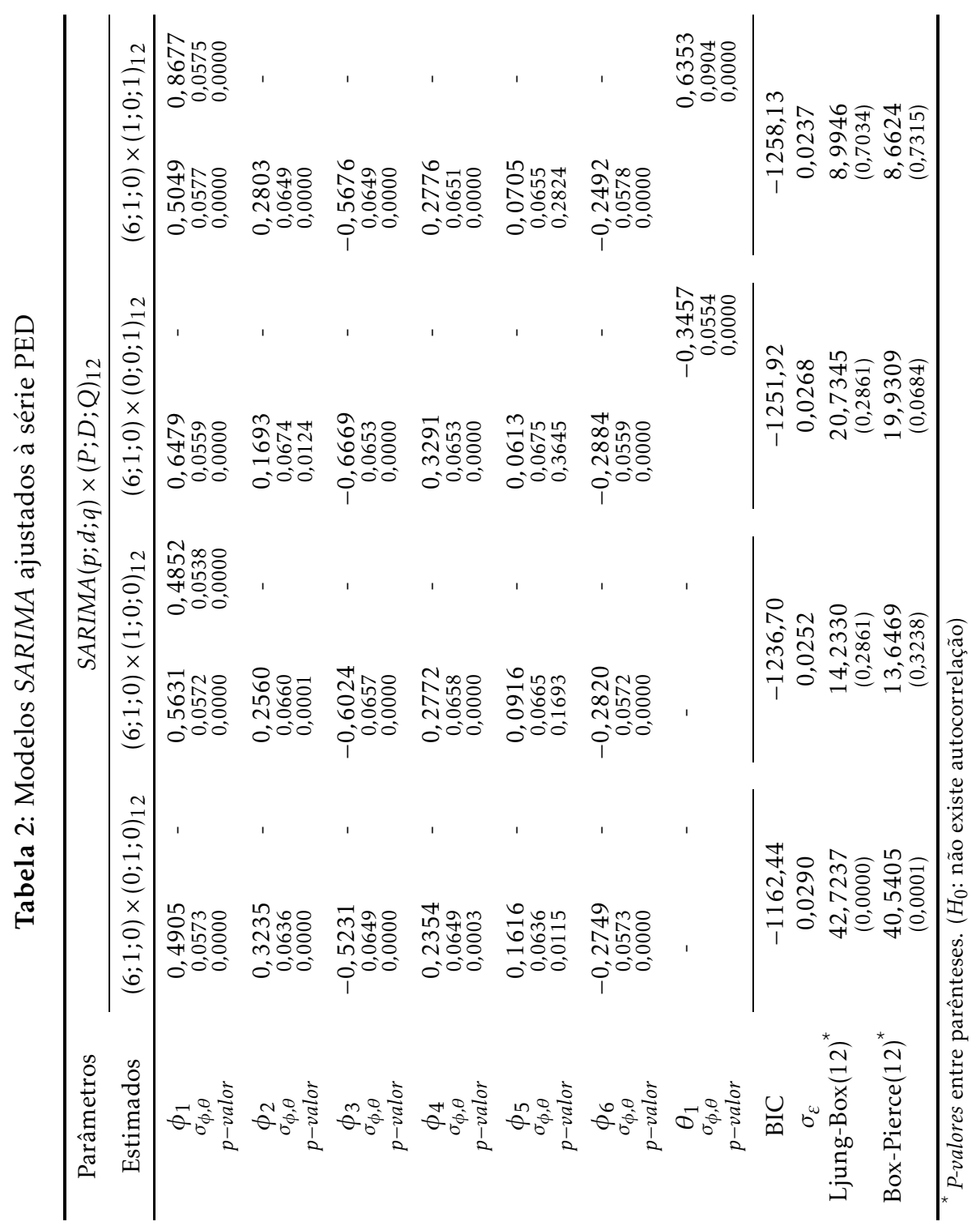




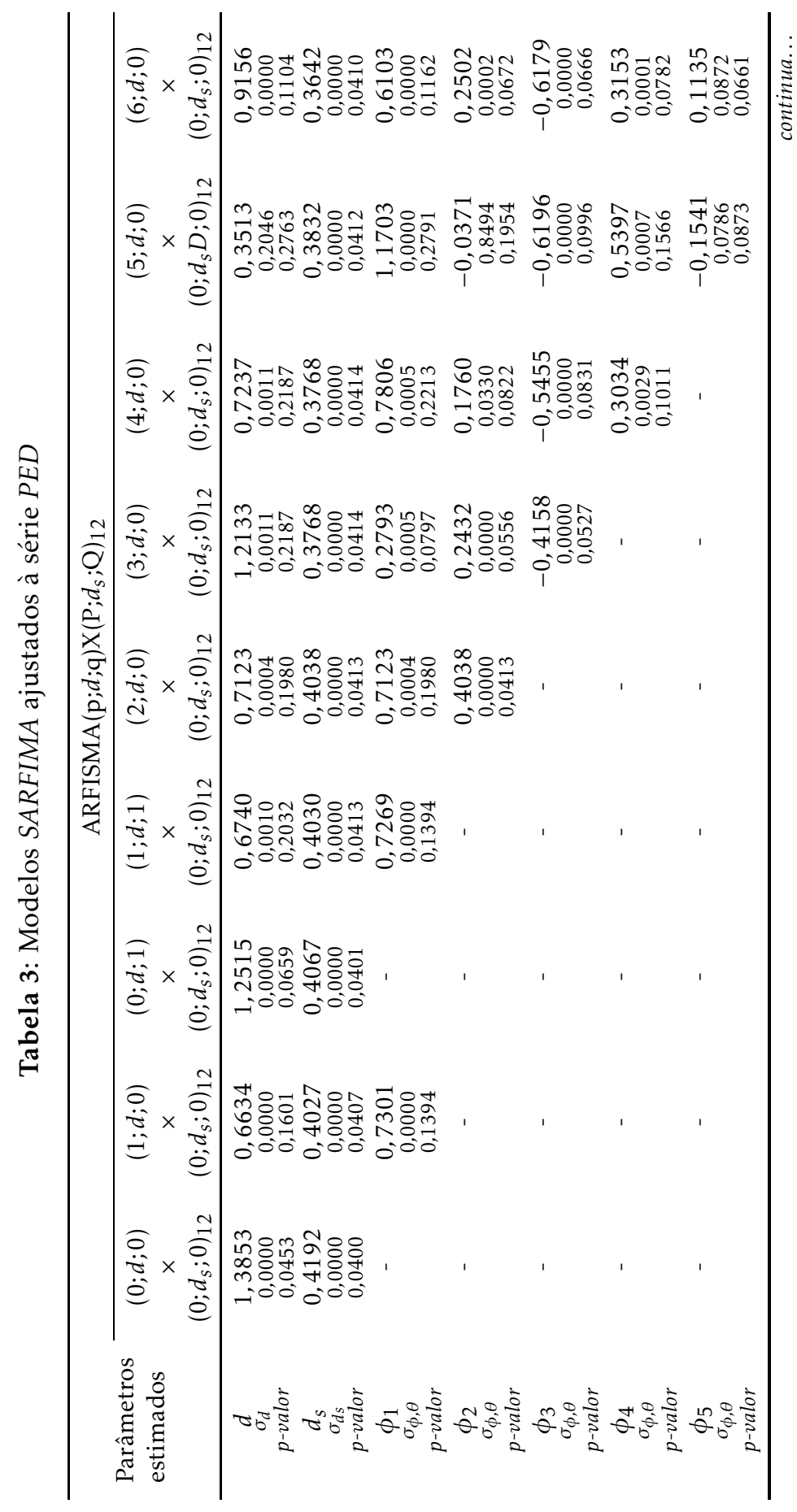




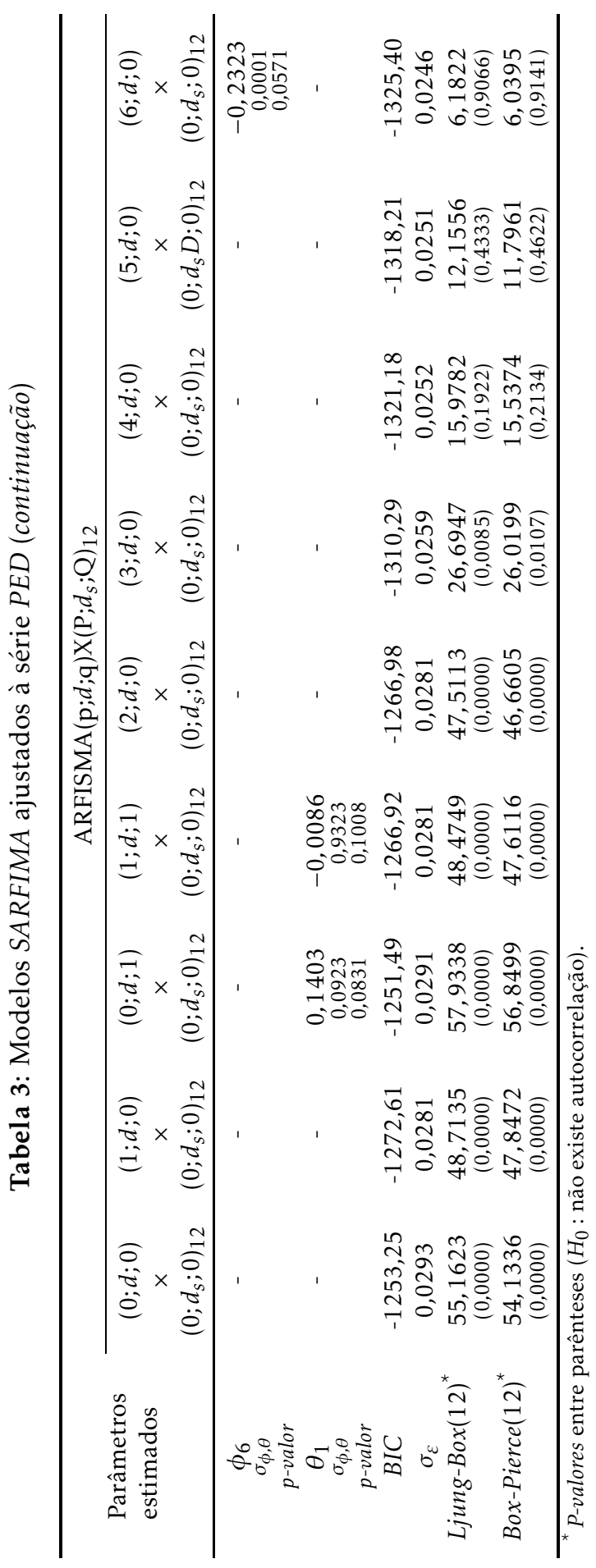


(a) PED Livre dos Componentes de Memória Longa

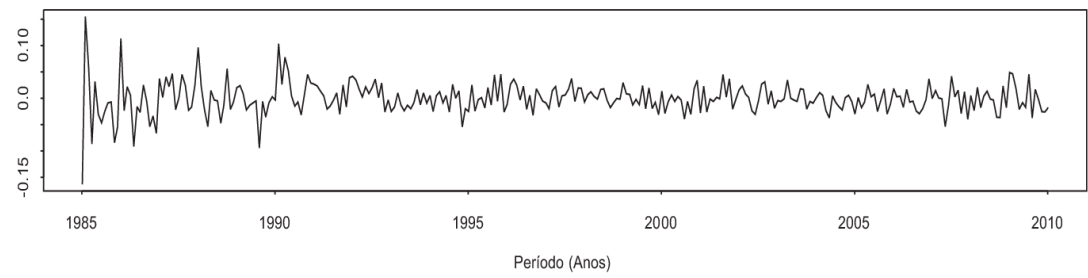

(b) Densidade Espectral da PED Livre dos Componentes de Memória Longa

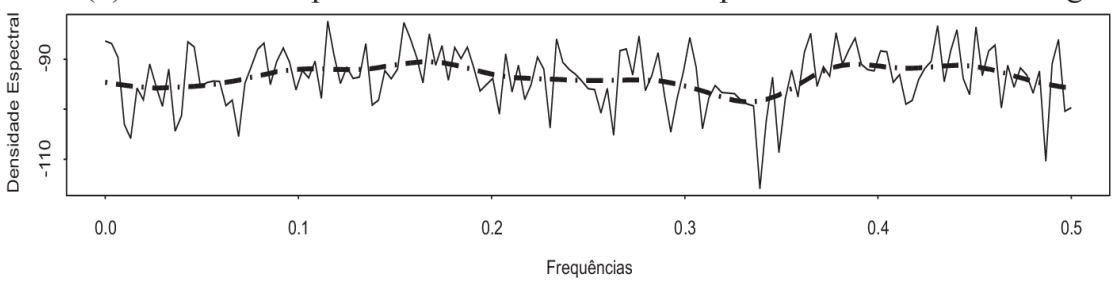

(b) FAC da PED Livre dos Componentes de Memória Longa

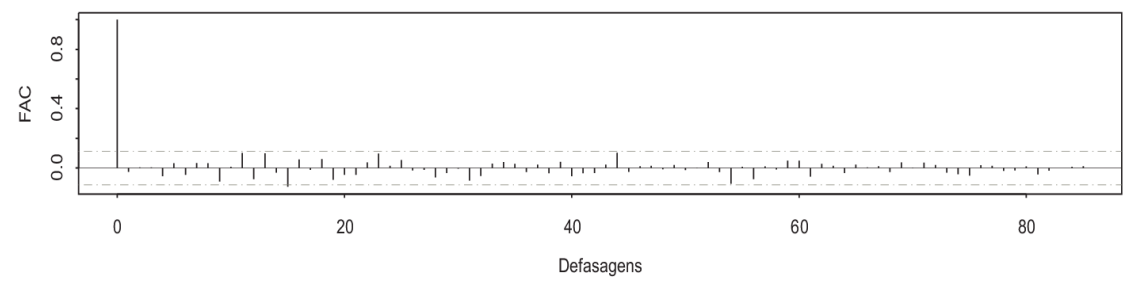

Figura 6: Série $P E D$ filtrada, seu espectro e $F A C$

de curto prazo. A FIR-S mostra como a trajetória da PED é afetada pelos choques estocásticos no longo prazo. Portanto, ela reflete como os choques no mercado de trabalho da Região Metropolitana de São Paulo são absorvidos pelo índice de desemprego medido pela $P E D$. Os modelos mostraram que a resposta do nível da série em função dos choques é determinada fundamentalmente pelo comportamento não estacionário da série e sofre grande influência do componente sazonal. A Tabela 4 mostra os valores estimados da FIR-S gerados pelos coeficientes dos modelos sazonais $\operatorname{SARIMA}(6 ; 1 ; 0) \times(1 ; 0 ; 1)_{12} \mathrm{e}$ $\operatorname{SARFIMA}(6 ; 0,9156 ; 0) \times(0 ; 0,3642 ; 0)_{12}$ para períodos entre 1 e 20 anos, enquanto a Figura 7 confronta graficamente suas trajetórias.

Os impactos dos choques sobre o nível da PED tendem a ser amplificados ao longo do tempo pelas duas abordagens, porém o modelo de memória longa mostra impactos bastante inferiores aos do modelo tradicional. No modelo de memória longa o caráter não estacionário é devido à interação entre os parâmetros de integração fracionária sazonal e não sazonal, enquanto no modelo SARIMA o caráter divergente da série deve-se à avaliação de uma raiz unitária na $P E D$. No curto prazo, os valores da FIR-S das duas abordagens apresentam-se relativamente próximos. Por exemplo, até 10 meses, tem-se: $\psi_{10}^{\text {SARIMA }}=1,3084$ e $\psi_{10}^{\text {SARFIMA }}=1,2675$. Isso indica que, no curto prazo, as previsões baseadas nos dois modelos não são discrepantes. Contudo, no médio e longo prazo seus valores divergem significativamente. O modelo 
Tabela 4: Função impulso-resposta sazonal da PED

\begin{tabular}{ccc}
\hline \multirow{2}{*}{$\begin{array}{c}\text { Meses à } \\
\text { Frente }\end{array}$} & SARIMA & FIR-S \\
\cline { 2 - 3 } & $(6 ; 1 ; 0) \times(1 ; 0 ; 1) 12$ & $(6 ; 0.9156 ; 0) \times(0 ; 0.3642 ; 0)_{12}$ \\
\hline 12 & 2,8485 & 1,6450 \\
24 & 4,7766 & 1,9994 \\
36 & 6,5291 & 2,2265 \\
48 & 8,0580 & 2,4031 \\
60 & 9,3846 & 2,5509 \\
120 & 13,8052 & 3,0783 \\
240 & 17,0490 & 3,7251 \\
\hline
\end{tabular}

FIR-S da $P E D$

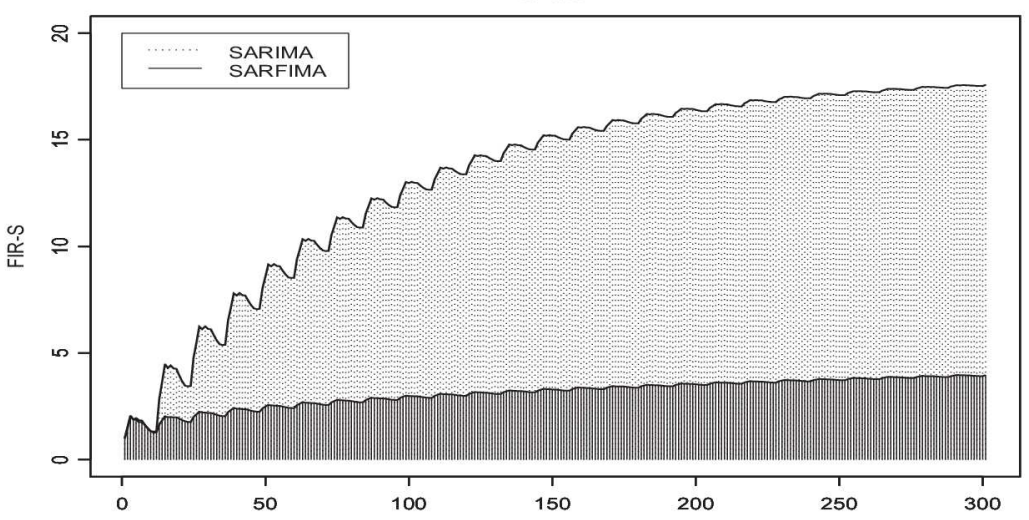

Figura 7: Função impulso-resposta sazonal do modelos $\operatorname{SARIMA}(6,1,0) \times$ $(1,0,1)_{12}$ vs. SARFIMA $(0,0.9156,0) \times(0,0.3642,0)_{12}$

SARIMA nitidamente superavalia os efeitos das inovações sobre o nível futuro da série, pois a diferenciação unitária ignora as informações contidas nas frequências próximas a zero. Os modelos de memória longa conseguem captar o comportamento associado às baixas frequências e, assim, produzem parâmetros mais adequados à representação do comportamento de longo prazo da série.

O caráter persistente não estacionário dos modelos de memória longa descarta a hipótese do nível da série desviar-se temporariamente de uma taxa de desemprego de longo prazo. Essa evidência não corrobora a hipótese de existência de uma taxa natural de desemprego, mostrando-se consistente com a hipótese de histerese na taxa de desemprego. Os efeitos dos choques que incidem na série são permanentemente incorporados em sua memória e amplificados ao longo do tempo. Os modelos prevêem que, após 10 anos, por exemplo, os efeitos das inovações na série serão amplificados em aproximadamente 3,0783 vezes e, após 20 anos, em 3,7251 vezes. ${ }^{9}$

\footnotetext{
${ }^{9}$ Devido à inexistência de uma série nacional de desemprego com um número observações suficiente para estimar os modelos de memória longa, torna-se impraticável a comparação da
} 
Existem diversos fatores que determinam o comportamento persistente percebido na série da $P E D$ da RMSP. Zylberstajn \& Balbinotto Netto (op. cit.) citam três razões básicas ligadas a questões institucionais que ajudam a explicar a histerese na taxa de desemprego na Europa na década de 1980 e que podem ajudar a compreender o comportamento na série brasileira. O primeiro motivo é a existência de direitos trabalhistas que punem os empregadores que desejam demitir seus trabalhadores. A relutância das firmas em contratar novos trabalhadores devido aos custos gerados por esse tipo de regulamentação no mercado de trabalho gera uma persistência no desemprego de longo prazo. Em segundo e terceiro lugar, estão os efeitos antagônicos gerados pela instituição do seguro-desemprego e do elevado grau de sindicalização. O seguro desemprego é apontado como fator que desestimula os desempregados a buscarem novos empregos e o elevado grau de sindicalização incentivaria os trabalhadores a buscarem salários mais elevados. Gray \& McDonald (2010) consideram que a sazonalidade no desemprego pode surgir como resultado da importância relativa de setores industriais mais afetados pelo fenômeno e também pelo baixo grau de instrução dos trabalhadores, que os tornam mais suscetíveis a contratações esporádicas. A influência sazonal na taxa do desemprego no caso da região metropolitana de São Paulo parece estar ligada à importância do setor de serviços na composição do produto interno. Nesse caso, o setor do comércio, por exemplo, no qual as contratações temporárias sofrem grande impulso no período de festas de fim de ano e dia das mães, pode ter grande influência na formação da sazonalidade no emprego. De acordo com dados do IBGE, o setor de serviços representou uma participação 69,0\% no total do PIB do estado de São Paulo em 2008, enquanto que os setores da indústria e agropecuária representaram, respectivamente, $29,5 \%$ e 1,4\%. ${ }^{10}$ Uma análise mais profunda sobre esses fatores e outras questões que implicam a persistência na taxa de desemprego necessitaria de pesquisa direcionada e, portanto, está fora do escopo desse trabalho. Para os próximos trabalhos vislumbra-se a realização de estudo comparando o fenômeno da persistência sazonal entre diferentes regiões metropolitanas brasileiras e entre outros países.

\section{Conclusões}

Esse trabalho retratou a persistência na série de desemprego da RMSP segundo as modelagens paramétricas circunscritas aos arcabouços $I(1)-I(0)$ e da integração fracionária. Foi utilizado um modelo de memória longa capaz de considerar as periodicidades estocásticas de natureza sazonal na série da $P E D$. Pelos dois arcabouços, fracionário e $I(1)-I(0)$, foi verificada a inconsistência na hipótese de convergência a uma taxa natural de desemprego na região analisada e, portanto, desfavorável à argumentação de uma taxa natural de

persistência na taxa de desemprego da região metropolitana de São Paulo com o desemprego total no Brasil.

${ }^{10}$ Estudos preliminares indicam que as regiões brasileiras em que o setor de serviços possui um maior peso na geração do produto, como o Distrito Federal $(93,3 \%)$ e Recife $(72,8 \%)$, por exemplo, exibem parâmetros de integração fracionária sazonal elevados nas regiões metropolitanas de suas capitais. Nos dois casos mencionados, os valores estimados são 0.3077 e 0,2664 , respectivamente. Para outras regiões metropolitanas de estados com menor importância do setor de serviços a dimensão do parâmetro tende a cair. No caso de Minas Gerais, por exemplo, a participação do setor de serviços é de $58,4 \%$ e o valor estimado do parâmetro de longa dependência sazonal para a região metropolitana de Belo Horizonte é 0,2301. 
desemprego. A trajetória da $P E D$ é essencialmente determinada por um comportamento não estacionário, coerente com a hipótese de histerese na taxa de desemprego criada para explicar o desemprego na Europa. A existência de um componente permanente na taxa de desemprego da $P E D$ exprime a elevada rigidez no mercado de trabalho da RMSP. Essa rigidez avaliada pelo modelo de memória longa sazonal revelou-se significativamente inferior àquela calculada pelo modelo tradicional sob o paradigma $I(1)-I(0)$. O estudo abre espaço para outras questões que poderão ser exploradas em pesquisas futuras, como a avaliação e comparação do grau de persistência sazonal na taxa de desemprego entre as diferentes regiões metropolitanas do país e em outros países que apresentam elevada influência sazonal em suas séries de desemprego, como Canadá, Espanha e Itália.

\section{Referências Bibliográficas}

Andel, J. (1986), 'Long memory time series models', Kybernetika 22(2), 251285.

Arteche, J. (2003), 'Semi-parametric robust tests on seasonal or cyclical long memory time series', Journal of Time Series Analysis 23, 251-285.

Arteche, J. \& Robinson, P. M. (2000), 'Semiparametric inference in seasonal and cyclicla long memory processes', Journal of Time Series Analysis 21, 1-25.

Ball, L. \& Mankiw, N. G. (2002), 'the nairu in theory and practice'. working paper 8940.', National Bureau of Economic Research. .

Beran, J. (1994), Statistics for Long-Memory Processes, Chapman \& Hall.

Bisognin, C. \& Lopes, S. R. (2007), 'Estimation and forecasting the long memory parameter in the presence of periodicity', Journal of Forecasting 26, 405427.

Bisognin, C. \& Lopes, S. R. (2009), 'Properties of seasonal long memory processes', Mathematical and Computer Modelling 49, 1837-1851.

Blanchard, O. J. \& Summers, L. H. (1986), Hysteresis and the european unemployment problem, Working Paper 1950, NBER.

Box, G. E. P. \& Jenkins, G. M. (1976), Time Series analysis: forecasting and control, revisited edn, Holden-Day, San Francisco.

Camarero, M. \& Tamarit, C. (2004), 'Hysteresis vs. natural rate of unemployment: new evidence for OECD countries', Economic Letters 84, 413-417.

Campbell, J. Y. S. R. J. (1987), 'Cointegration and test of present value models', Journal of Political Economy 95, 1062-1088.

Chung, C. (1996), 'A generalized fractionlly integrated autoregressive moving-average precess', Journal of Time Series Analysis 17(2), 111-140.

Clement, J., L., L. \& Montanés, A. (2005), 'The unemployment structure of the US states', The Quarterly Review of Economics and Finance 45, 848-868. 
Dickey, D. A. \& Pantula, S. G. (1987), 'Determining the order of differencing in autoregressive processes', Journal of Business and Economic Statistics 5(4), 455-461.

Dickey, D. \& Fuller, W. (1979), 'Distribution of the estimators for autoregressive time series with a unit root.', Journal of the American Statistical Association 74(366), 427-731.

Diebold, F. X. \& Rudebush, G. D. (1989), 'Long memory and persistence in aggregate output', Journal of Monetary Economics 24, 189-209.

Diebold, F. X. \& Rudebush, G. D. (1991), 'On the power of Dickey-Fuller tests against fractional alternatives', Economic Letters 35, 155-160.

Diongue, A. K., Diop, A. \& Ndongo, M. (2008), 'Seasonal fractional ARIMA with stable innovations', Statistics and probability letters 78, 1404-1411.

Engle, R. F. \& Granger, C. W. J. (1987), 'Co-integration and error correction representation, estimation, and testing', Econometrica 55(2), 251-276.

Fava, V. L. \& Alves, D. C. O. (1998), 'Longa persistência nas taxas de inflação', Revista de Econometria 18(2), 245-264.

Fernandes, R. (1998), 'Encargos sociais e demanda por trabalho no setor formal da economia', Revista de Economia Aplicada 2(3), 553-578.

Fernandes, R. \& Cacciamali, M. C. (1993), 'Distribuição dos trabalhadores e diferenciais de salários entre mercado de trabalho regulamentado e nãoregulamentado', Estudos Econômicos 23.

Fernandes, R. \& Chahad, J. P. Z. (2002), 'Unemployment insurance and transitions in the labor market: an evaluation of the brazilian program', Brazilian Review of Econometrics 22.

Friedman, M. (1968), 'The role of monetary policy', American Economic Review $58,1-18$.

Geweke, J. \& Porter-Hudak, S. (1983), 'The estimation and applications of the long memory time series modesls', Journal of Time Series Analysis 4(4).

Gil-Alana, L. A. (2007), 'Long run an cyclical strong dependence in macroeconomic time series: Nelson and Plosses revisited', Empirica 34, 139-154.

Gil-Alana, L. A. (2008), 'Testing of seasonal integration and cointegration with fractionally integrated techniques: an application to the danish labour demand', Economic Modelling 25, 326-339.

Giraitis, L. \& Leipus, R. (1995), 'A generalized fractionally differencing approach in longo memory modelling', Lithuanian Mathematical Journal 35, 6581 .

Gomes, F. A. R. \& Gomes da Silva, C. (2009), 'Hyteresis vs NAIRU and convergence vs divergence: the behavior of regional unemployment rates in Brazil', The Quarterly Review of Economics and Finance 49, 308-322.

Granger, C. W. J. (1966), 'The typical spectral shape of an economic variable', Econometrica 34, 150-161. 
Granger, C. W. J. \& Joyeux, R. (1980), 'An introduction to long-memory time series models and fractional differencing', Journal of Time Series Analysis $1,15-29$.

Gray, D. M. \& McDonald, J. T. (2010), 'Seasonal employment in Canada: its decline and its persistence', Canadian Public Policy 36(1), 1-27.

Gray, H. L., Zhang, N. F. \& Woodward, W. A. (1989), 'On gereralized fractional precesses', Journal of Time Series Analysis 10, 233-257.

Hassler, U. \& Wolters, J. (1994), 'On the power of unit root tests against fractional alternatives', Economics Letters 45, 1-5.

Hosking, J. R. M. (1981), 'Fractional differencing', Biometrika 68, 165-176.

Hsu, N. \& Tsai, H. (2009), 'Semiparametric estimation for seasonal llongmemory time series using generalized exponential models', Journal of Statistical Planning and Inference 139.

Johansen, S. (1995), Likelihood bases inference in cointegrated vector error correction, Oxford University Press, Oxford.

Kwiatkowski, D., Phillips, P. C. B., Schmidt, P. \& Shin, Y. (1992), 'Testing the null hypothesis of stationary against the alternative of a unit root', Journal of Econometrics 54, 159-178.

Lee, J. W. (1996), 'Government interventions and productivity growth', Journal of Economic Growth 1, 391-414.

Nelson, C. \& Plosser, C. (1982), 'Trends and random walks in macroeconomics time series', Journal of Monetary Economics 10(3), 139-162.

Ooms, M. (1995), Flexible seasonal long memory and economic time series, Technical Report 9515/A, Econometric Institute, Erasmus University.

Palma, W. (2007), Long-Memory time series: theory and methods, Wiley.

Phillips, P. C. B. \& Perron, P. (1988), 'Testing for a unit root in time series regression', Biometrika 75(2), 335-346.

Porter-Hudak1990 (1990), 'An application of seasonal fractionally differenced model to the monetary aggregate', Journal of American Statistical Association 85, 338-344.

Reisen, V. A., Cribari-Neto, F. \& Jensen, M. (2003), 'Lonto memory dynamics: the case of Brazil', Studies in Nonlinear Dynamics and Econometrics 7(3).

Reisen, V. A., Rodrigues, A. L. \& Palma, W. (2006), 'Estimation of seasonal fractionally integrated process', Computational Statistics E Data Analysis 50, 568-582.

Said, S. E. \& Dickey, D. (1984), 'Testing for unit roots in autoregressive moving-average models with unknown order', Biometrika 71, 599-607.

Smith, S. W. (1994), Labor Economics, Routhledge. 
Song, F. \& Wu, Y. (1998), 'Hysteresis in unemployment: evidence from OECD countries', The Quarterly Review of Economics and Finance 38(2), 181-192.

Ulissea, G. (2008), 'Instituições e a informalidade no mercado de trabalho', Estudos Econômicos 38(3), 87-99.

Ulissea, G. (2010), 'Regulation of entry, labor market institutions and the informal sector', Journal of Development Economics 91, 87-99.

Woodward, W. A., Cheng, G. C. \& Gray, H. L. (1998), 'A k-factor GARMA long-memory model', Journal of Time Series Analysis 19(5), 485-504.

Zylberstajn, H. \& Balbinoto Neto, G. (1999), 'As teorias de desemprego e políticas públicas de emprego', Estudos Econômicos 39(1), 129-149. 